\title{
Physical Activity, Screen Time, and Sleep Duration of Children Aged 6-9 Years in 25 Countries: An Analysis within the WHO European Childhood Obesity Surveillance Initiative (COSI) 2015-2017
}

\author{
Stephen Whitinga, b Marta Buoncristiano ${ }^{a}$ Peter Gelius ${ }^{c}$ Karim Abu-Omar ${ }^{c}$ Mary Pattison ${ }^{a}$ \\ Jolanda Hyskad Vesselka Duleva ${ }^{\text {e }}$ Sanja Musić Milanović ${ }^{\text {Hana Zamrazilovág }}$ \\ Tatjana Hejgaard $^{\text {h }}$ Mette Rasmussen ${ }^{i}$ Eha Nurk ${ }^{j} \quad$ Lela Shengeliak Cecily C. Kelleher $^{\mathrm{l}}$ \\ Mirjam M. Heinen' ${ }^{\prime}$ Angela Spinelli ${ }^{m}$ Paola Nardone ${ }^{m}$ Akbota Abildina $^{n}$ \\ Shynar Abdrakhmanova ${ }^{\text {n }}$ Gulmira Aitmurzaeva ${ }^{\circ}$ Zhamyila Usuopva $^{\circ}$ Iveta Pudule ${ }^{p}$ \\ Aušra Petrauskiene $^{q}$ Victoria Farrugia Sant'Angelo ${ }^{r}$ Enisa Kujundzic ${ }^{s}$ Stevo Popovic ${ }^{t}$ \\ Anne-Siri Fismen ${ }^{u}$ Ingunn Holden Bergh ${ }^{v}$ Anna Fijalkowskaw ${ }^{w}$ Ana Isabel Rito ${ }^{x}$ \\ Alexandra Cucu ${ }^{y}$ Lacramioara Aurelia Brinduse ${ }^{z}$ Valentina Peterkova ${ }^{A}$ Andrea Gualtieri ${ }^{B}$ \\ Marta García-Solano ${ }^{C}$ Enrique Gutiérrez-González ${ }^{C}$ Zulfinissio Abdurrahmonova ${ }^{D}$ \\ Khadichamo Boymatova ${ }^{\mathrm{E}}$ Nazan Yardim ${ }^{\mathrm{F}}$ Maya Tanrygulyyeva ${ }^{\mathrm{G}}$ Daniel Weghuber $^{\mathrm{H}}$ \\ Karin Schindler' Dragana Stojisavljević J Aida Filipović Hadžiomeragić, ${ }^{\mathrm{K}}$ \\ Eliza Markidou lonnaiduL ${ }^{L}$ Wolfgang Ahrens ${ }^{\mathrm{M}}$ Maria Hassapidou ${ }^{\mathrm{N}}$ Viktoria Anna Kovacs $^{\mathrm{O}}$ \\ Sergej M. Ostojic ${ }^{\mathrm{P}} \quad$ Lubica Ticha ${ }^{\mathrm{Q}}$ Gregor Starc ${ }^{\mathrm{R}}$ Kenisha Russell Jonsson ${ }^{\mathrm{S}}$ Igor Spiroski $^{\top}$ \\ Harry Rutter ${ }^{\mathrm{U}}$ Romeu Mendes ${ }^{\mathrm{a}, \mathrm{b}}$ Julianne Williams ${ }^{\mathrm{a}} \quad$ Ivo Rakovac $^{\mathrm{a}} \quad$ João Breda ${ }^{\mathrm{a}}$
}

${ }^{a}$ WHO European Office for the Prevention and Control of NCDs, Moscow, Russian Federation; ${ }^{b}$ EPIUnit - Instituto de Saúde Pública, Universidade do Porto, Porto, Portugal; ' ${ }^{\circ}$ epeprtment of Sport Science and Sport, FAU, Erlangen,

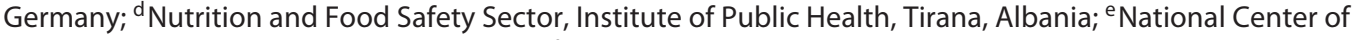
Public Health and Analyses, Sofia, Bulgaria; ${ }^{f}$ School of Medicine, University of Zagreb, Croatian Institute of Public Health, Zagreb, Croatia; IInstitute of Endocrinology, Obesity Management Centre, Prague, Czechia; ${ }^{\mathrm{h}}$ Danish Health Authority, Copenhagen, Denmark; 'National Institute of Public Health, University of Southern Denmark, Copenhagen, Denmark; ${ }^{j}$ National Institute for Health Development, Tallinn, Estonia; ${ }^{k}$ National Center for Disease Control and Public Health, Tbilisi, Georgia; 'National Nutrition Surveillance Centre, University College Dublin, Dublin, Ireland; ${ }^{\mathrm{m}}$ Italian National Institute of Health, Rome, Italy; ${ }^{\mathrm{n}}$ National Centre of Public Health of the Ministry of Health of the Republic of Kazakhstan, Nur-Sultan, Kazakhstan; ${ }^{\circ}$ Republican Centre for Health Promotion, Bishkek,

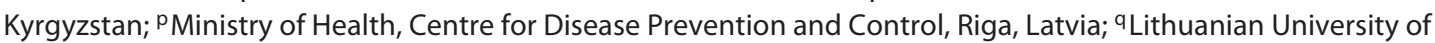
Health Sciences, Health Research Institute and Department of Preventive Medicine, Kaunas, Lithuania; ${ }^{r}$ Primary Health Care, Floriana, Malta; ${ }^{\mathrm{s}}$ Institute of Public Health of Montenegro, Podgorica, Montenegro; ${ }^{\mathrm{t}}$ Faculty for Sport and Physical Education, University of Montenegro, Niksic, Montenegro; "Department of Health Promotion, Norwegian Institute of Public Health, Bergen, Norway; ${ }^{\vee}$ Department of Health and Inequality, Division of Mental

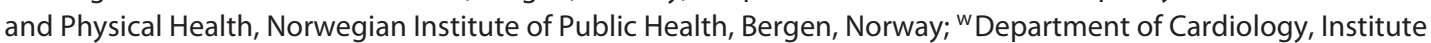
of Mother and Child, Warsaw, Poland; ${ }^{x}$ National Institute of Health Dr Ricardo Jorge I.P., Lisbon, Portugal; ${ }^{y}$ National Institute of Public Health, Bucharest, Romania; ${ }^{2}$ University of Medicine and Pharmacy Carol Davila, Bucharest, Romania;

(C) 2020 The Author(s)

Published by S. Karger AG, Basel

This is an Open Access article licensed under the Creative Commons Attribution-NonCommercial-4.0 International License (CC BY-NC) (http://www.karger.com/Services/OpenAccessLicense), applicable to the online version of the article only. Usage and distribution for commercial purposes requires written permission.
Stephen Whiting

WHO European Office for the Prevention and Control of Noncommunicable Diseases WHO Regional Office for Europe

Leontievsky Per. 9, Moscow 125009 (Russian Federation) whitings@who.int 


\begin{abstract}
${ }^{A}$ The Endocrine Research Centre, Moscow, Russian Federation; ${ }^{B}$ Health Authority San Marino, San Marino, Republic of San Marino; ${ }^{C}$ Spanish Agency for Food Safety and Nutrition, Ministry of Health, Madrid, Spain; ${ }^{D}$ Republican Centre for Nutrition, Ministry of Health and Social Protection of Population, Duschanbe, Tajikistan; ${ }^{E}$ WHO Tajikistan

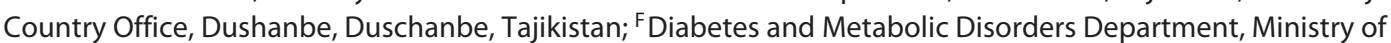
Health, Public Health Institution, Ankara, Turkey; ${ }^{G}$ Scientific Research Institute of Maternal and Child Health, Ashgabat, Turkmenistan; ${ }^{H}$ Department of Pediatrics, Paracelsus Medical University, Salzburg, Austria; 'Medical University of Vienna, Vienna, Austria; JPublic Health Institute of Republic of Srpska, the University of Banja Luka, Faculty of Medicine, Banja Luka, Bosnia and Herzegovina; ${ }^{K}$ Institute of Public Health of Federation of Bosnia and Herzegovina, Banja Luka, Bosnia and Herzegovina; 'Ministry of Health, Limassol, Cyprus; ${ }^{M}$ Leibniz Institute for Prevention Research and Epidemiology - BIPS, Bremen, Institute of Statistics, University of Bremen, Bremen, Germany; ${ }^{\mathrm{N}}$ International Hellenic University, Thessaloniki, Greece; ${ }^{\circ}$ Hungarian School Sport Federation, Budapest, Hungary; ${ }^{P}$ Faculty of Sport and PE, University of Novi Sad, Novi Sad, Serbia; ${ }^{Q}$ National Institute of Children Diseases, Medical Faculty of Comenius University, Bratislava, Slovakia; ${ }^{2}$ Faculty of Sport, University of Ljubljana, Ljubljana, Slovenia; ${ }^{S}$ Department of Living Conditions and Lifestyle, Public Health Agency of Sweden, Solna, Sweden; ${ }^{\top}$ Department of Physiology and Monitoring of Nutrition, Institute of Public Health, Skopje, Republic of Macedonia;

UDepartment of Social and Policy Sciences, University of Bath, Bath, UK
\end{abstract}

\section{Keywords}

Physical inactivity $\cdot$ Surveillance $\cdot$ Sedentary behaviour . Active transport, active play

\begin{abstract}
Background: Children are becoming less physically active as opportunities for safe active play, recreational activities, and active transport decrease. At the same time, sedentary screen-based activities both during school and leisure time are increasing. Objectives: This study aimed to evaluate physical activity (PA), screen time, and sleep duration of girls and boys aged 6-9 years in Europe using data from the WHO European Childhood Obesity Surveillance Initiative (COSI). Method: The fourth COSI data collection round was conducted in 2015-2017, using a standardized protocol that included a family form completed by parents with specific questions about their children's PA, screen time, and sleep duration. Results: Nationally representative data from 25 countries was included and information on the PA behaviour, screen time, and sleep duration of 150,651 children was analysed. Pooled analysis showed that: $79.4 \%$ were actively playing for $>1 \mathrm{~h}$ each day, $53.9 \%$ were not members of a sport or dancing club, $50.0 \%$ walked or cycled to school each day, $60.2 \%$ engaged in screen time for $<2 \mathrm{~h} /$ day, and $84.9 \%$ slept for 9-11 h/night. Country-specific analyses of these behaviours showed pronounced differences, with national prevalences in the range of $61.7-98.3 \%$ actively playing for $>1 \mathrm{~h} /$ day, $8.2-85.6 \%$ were not members of a sport or dancing club, 17.7-94.0\% walked or cycled to school each day, 32.3$80.0 \%$ engaged in screen time for $<2 \mathrm{~h} /$ day, and $50.0-95.8 \%$
\end{abstract}

slept for 9-11 h/night. Conclusions: The prevalence of engagement in PA and the achievement of healthy screen time and sleep duration are heterogenous across the region. Policymakers and other stakeholders, including school administrators and parents, should increase opportunities for young people to participate in daily PA as well as explore solutions to address excessive screen time and short sleep duration to improve the overall physical and mental health and well-being of children.

(C) 2020 The Author(s)

Published by S. Karger AG, Basel

\section{Background}

During childhood, adequate levels of physical activity (PA) are fundamental for the development of basic cognitive, motor, and social skills, as well as musculoskeletal, cardiovascular, and metabolic health [1]. PA is an important determinant in the prevention and treatment of childhood obesity and early metabolic risk factors [2, 3]. Childhood obesity is associated with many serious health problems $[4,5]$ including psychosocial consequences [69] and it increases the risk for noncommunicable diseases (NCDs) later in life [10]. PA levels during childhood also tend to track into adolescence and adulthood [10, 11 ], so establishing healthy PA behaviours during childhood can reap dividends in later life [12].

The World Health Organisation (WHO), through the Global Action Plan for Physical Activity 2018-2030 [13] and the Physical Activity Strategy for the WHO European 
Region 2016-2025 [14], provides guidance and support for countries to promote regular PA as a preventative measure to help reduce a wide variety of health risk factors across all ages, genders, ethnicities, and socioeconomic subgroups. Current WHO recommendations for children and adolescents aged 5-17 years are to accumulate at least $60 \mathrm{~min}$ of moderate/vigorous-intensity PA (MVPA) per day to maintain good physical and mental health and well-being [1]. Active play (unstructured, outdoor physical activity in children's free time), active transport (cycling or walking) and participating in sports are the major contributors to total PA among children.

It is important that, as children grow and develop, in addition to high levels of PA, they also achieve low levels of sedentary behaviour (SB) and get enough sleep each day. Time spent using screen-based devices increases SB which has been linked with a higher intake of energydense snacks and drinks and fast foods, and an overall higher energy intake [15]. Short sleep duration has also been linked with the development of obesity [16] and high blood pressure in children [17] as well as with overall well-being [18]. In recent years and based on evidence of the importance of SB and sleep to health, several countries have shifted to providing integrated guidance for movement behaviours across the whole day (i.e., a 24-h period) including recommendations for PA, screen time, and sleep duration [19-21].

The COSI (Childhood Obesity Surveillance Initiative) system is unique as it involves taking standardised weight and height measurements of children, providing nationally representative data for participating countries and a large region-wide dataset for analysing the determinants of childhood overweight and obesity. It includes a questionnaire that measures the PA, screen time, and sleep duration of children, a voluntary element for the participating countries, which is completed by a child's family member (usually a parent). Comparable data on PA-related behaviours among children in Europe is limited as countries use a range of approaches, including parent/ caregiver report measures (e.g., questionnaires) and objective measures (e.g., accelerometers). While there are weaknesses to using parent/caregiver report questionnaires, one of their strengths is the collection of a wide range of sociodemographic and health-related behaviours of study participants by means of a single tool from large nationally representative samples. Objective measures of PA have thus far not been integrated with the COSI system due to the costs involved for the participating countries as well as the added complication to the implementation of a survey where the primary outcome is nationally representative data on childhood overweight and obesity; information on PA, screen time, and sleep are just 3 of many secondary outcomes.

This study aimed to describe overall and cross-national comparisons of PA, screen time, and sleep duration of girls and boys aged 6-9 years using data collected as part of the COSI study in 2015-2017 in 25 countries of the WHO European Region. An analysis of this unique dataset provides new insights and includes many countries where there is currently limited data on these behaviours.

\section{Methods}

The fourth COSI (COSI round 4) data collection was conducted in 36 countries in the school years 2015/2016 and 2016/2017 (2017/2018 for Kyrgyzstan). Information on children's PA, screen time, and sleep duration was collected via a paper questionnaire (i.e., the COSI family form) that was completed by a parent or caregiver [22]. Use of the family form is an optional part of the study and, of the 36 countries that participated, 24 included it in their national protocol which provided the data used in this study. One additional country, Estonia, did not use the family form but collected data by asking the children directly. The Estonian data is thus presented here but was not included in the pooled estimates.

\section{Study Design and Sampling}

The data collection followed a standard protocol developed in 2007 by the WHO Regional Office for Europe, together with its member states, which was then slightly amended for the COSI rounds 2, 3, and 4 [23-26]. The COSI data collections have followed the International Ethics Guidelines for Biomedical Research Involving Human Subjects [27] and national ethics committees approved all national study protocols.

According to the COSI protocol, countries could choose $\geq 1$ of the following age groups: $6.0-6.9,7.0-7.9,8.0-8.9$, and 9.0-9.9 years. Most of the countries targeted only the 7 -year-olds, or also $\geq 1$ other age group. In almost all countries, the participating children were enrolled in primary school and were then randomly selected to create a nationally representative sample. More details on the main characteristics of the study design in each country are provided in the online supplementary Table 1 (for all online suppl. material, see www.karger.com/doi/10.1159/000511263) and elsewhere [28].

\section{Data Elaboration}

Information from the COSI family form included the following 5 behaviours: transportation to and from school, membership of a sport or dancing club and time spent practising these, time spent actively playing, time spent watching TV or using electronic devices, and the number of hours of sleep per night.

Table 1 shows the questions of interest and their predefined answer options for each of the categorical variables included in the analysis. In some cases, answer options were collapsed into broader categories to facilitate comparisons between countries. The WHO Regional Office for Europe collected data from all countries using a common format and carried out a data-cleaning process to 


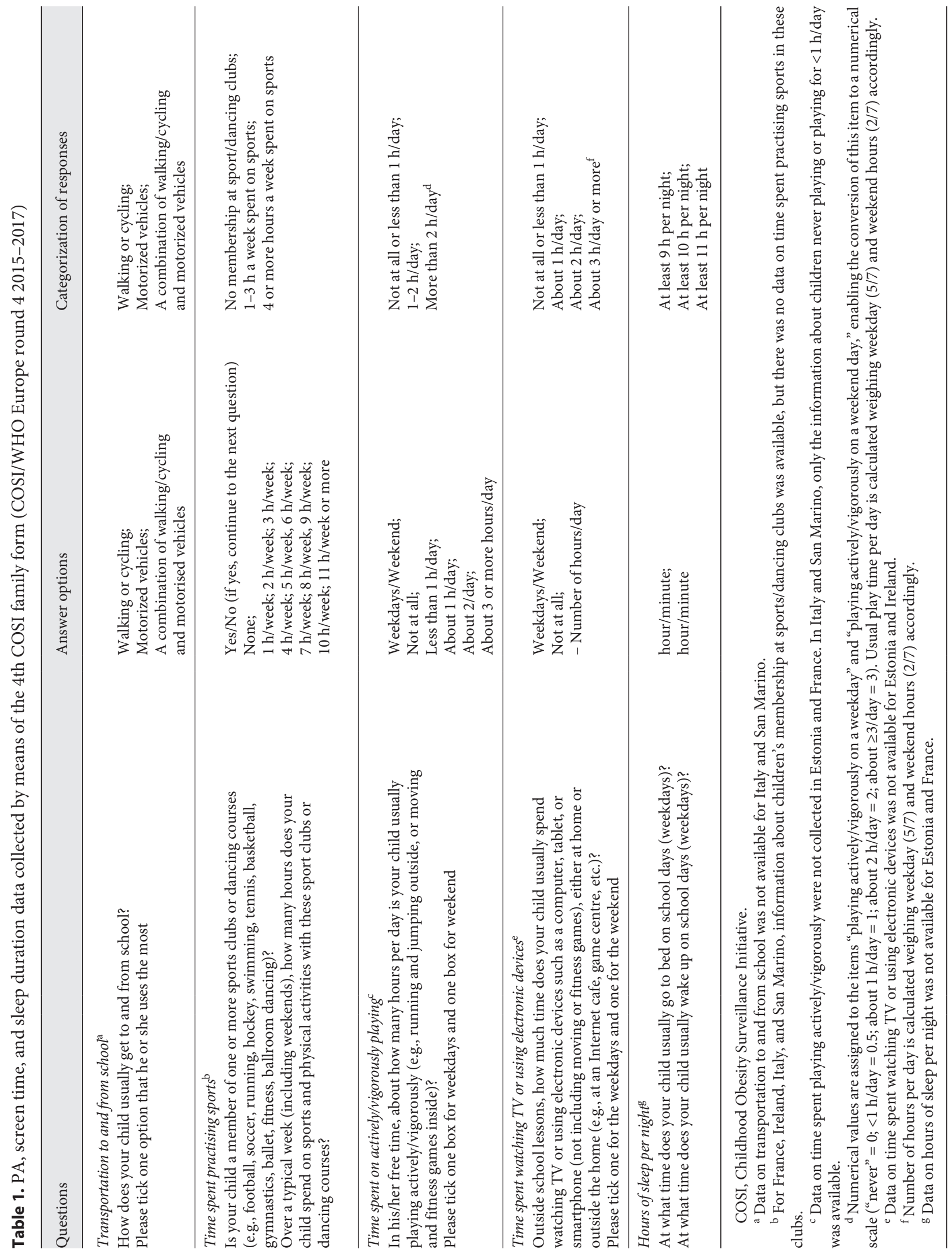


Table 2. The number of children invited to participate in COSI/WHO Europe round 4 2015/2017, the proportions whose parents completed the family forms, the number of children included in the final analysis, and their demographic characteristics by country

\begin{tabular}{|c|c|c|c|c|c|c|c|c|c|c|}
\hline \multirow[t]{2}{*}{ Country $^{\mathrm{a}}$} & \multicolumn{2}{|c|}{ Children invited to participate ${ }^{b}$} & \multicolumn{3}{|c|}{ Children included in the analysis $^{c}$} & \multicolumn{5}{|c|}{ Distribution of children by sex and age ${ }^{d}, \%$} \\
\hline & Total, $n$ & $\begin{array}{l}\text { family form } \\
\text { completed, \% }\end{array}$ & boys, $n$ & girls, $n$ & total, $n$ & boys & 6 years & 7 years & 8 years & 9 years \\
\hline ALB & 7,113 & 36.2 & 1,315 & 1,212 & 2,527 & 52.5 & 0.1 & 24.2 & 52.0 & 23.7 \\
\hline BUL & 4,090 & 83.1 & 1,702 & 1,698 & 3,400 & 51.5 & 0.0 & 100 & 0.0 & 0.0 \\
\hline $\mathrm{CRO}^{\mathrm{e}}$ & 7,220 & 76.0 & 1,318 & 1,333 & 2,651 & 51.1 & 0.0 & 0.0 & 100 & 0.0 \\
\hline $\mathrm{CZH}$ & n.a. & n.a. & 670 & 736 & 1,406 & 50.7 & 49.5 & 50.5 & 0.0 & 0.0 \\
\hline DEN & 3,202 & 29.9 & 511 & 446 & 957 & 52.7 & 27.4 & 70.2 & 2.4 & 0.0 \\
\hline EST & 14,038 & 91.9 & 6,598 & 6,246 & 12,844 & 51.4 & 0.2 & 48.9 & 49.8 & 1.1 \\
\hline FRA & 7,094 & 75.6 & 2,649 & 2,669 & 5,318 & 50.0 & 5.5 & 36.6 & 43.7 & 14.1 \\
\hline GEO & 4,143 & 78.4 & 1,667 & 1,579 & 3,246 & 51.2 & 1.6 & 85.1 & 13 & 0.3 \\
\hline IRE & 2,704 & 32.4 & 438 & 436 & 874 & 52.6 & 38.2 & 60.2 & 1.6 & 0.0 \\
\hline ITA & 50,902 & 95.2 & 22,425 & 21,271 & 43,696 & 51.5 & 0.0 & 0.6 & 66.3 & 33.1 \\
\hline KAZ & 6,026 & 82.3 & 2,149 & 2,162 & 4,311 & 50.6 & 0.0 & 0.4 & 51 & 48.6 \\
\hline KGZ & 8,773 & 86.6 & 3,798 & 3,769 & 7,567 & 50.7 & 10.5 & 43.5 & 39.5 & 6.5 \\
\hline LTU & 5,527 & 69.8 & 1,930 & 1,882 & 3,812 & 50.6 & 0.4 & 66.4 & 33 & 0.2 \\
\hline LVA & 8,143 & 71.5 & 2,752 & 2,955 & 5,707 & 48.2 & 7.9 & 43.8 & 9.1 & 39.3 \\
\hline MAT & 4,329 & 73.4 & 1,589 & 1,590 & 3,179 & 50.0 & 0.1 & 69.7 & 30.1 & 0.1 \\
\hline MNE & 4,094 & 66.8 & 1,441 & 1,295 & 2,736 & 52.8 & 31.2 & 48.4 & 20.1 & 0.2 \\
\hline POL & 3,828 & 76.9 & 1,451 & 1,494 & 2,945 & 50.2 & 0.0 & 0.0 & 100.0 & 0.0 \\
\hline POR & 7,475 & 85.6 & 3,167 & 3,224 & 6,391 & 50.7 & 25.2 & 49.0 & 24.0 & 1.7 \\
\hline ROM & 9,094 & 73.6 & 3,312 & 3,298 & 6,610 & 49.1 & 0.4 & 28.4 & 47.5 & 23.8 \\
\hline RUS & 3,900 & 52.6 & 1,006 & 1,046 & 2,052 & 50.2 & 18.8 & 72.8 & 8.3 & 0.1 \\
\hline SMR & 329 & 93.6 & 138 & 168 & 306 & 45.1 & 0.0 & 0.0 & 64.7 & 35.3 \\
\hline SPA & 14,908 & 70.1 & 5,290 & 5,163 & 10,453 & 50.9 & 25.4 & 25.2 & 24.9 & 24.6 \\
\hline TJK & 3,502 & 93.5 & 1,623 & 1,647 & 3,270 & 51.6 & 7.7 & 90.8 & 1.4 & 0.2 \\
\hline TKM & 4,085 & 95.3 & 1,944 & 1,947 & 3,891 & 49.9 & 0.0 & 79.9 & 20.1 & 0.0 \\
\hline TUR & 14,164 & 81.7 & 5,335 & 5,167 & 10,502 & 50.9 & 11.4 & 82.3 & 6.0 & 0.3 \\
\hline Total & 198,683 & 79.5 & 76,218 & 74,433 & 150,651 & 51,3 & 0.0 & 45.7 & 48.6 & 5.7 \\
\hline
\end{tabular}

n.a., not available.

${ }^{a}$ Figures refer to primary school children from: Albania (ALB); Bulgaria (BUL); Croatia (CRO); Czechia (CZH); Denmark (DEN); France (FRA) Georgia (GEO); Italy (ITA); Kazakhstan (KAZ); Kyrgyzstan (KGZ); Lithuania (LTU); Latvia (LVA); Malta (MAT); Montenegro (MNE); Poland (POL); Portugal (POR); Romania (ROM); Moscow city (RUS); San Marino (SMR); Spain (SPA); Tajikistan (TJK); Turkmenistan (TKM) and Turkey (TUR).

$\mathrm{b}$ Total figures were calculated including only countries with available information about the number of children invited to participate in the surveillance. The Estonian percentage refers to the proportion of child's form filled in, as the family form was not used in the 4th round of COSI and questions about PA patterns were put to children directly, not to parents/caregivers.

${ }^{c}$ All children with complete information on sex, aged 6-9 years, and with information about physical activity, sedentary behaviour, and sleep duration from the family form.

${ }^{\mathrm{d}}$ Pooled values were estimated including the following age groups/countries: 7-year-olds from Bulgaria, Czechia, Denmark, Estonia, Kyrgyzstan, Georgia, Ireland, Latvia, Lithuania, Malta, Montenegro, Portugal, Spain, Tajikistan, Turkey, and Turkmenistan; 8-year-olds from Albania, Croatia, Italy, France, Poland, Romania, and San Marino; and 9-year-olds from Kazakhstan. The figures were estimated by applying post-stratification weights.

e For Croatia, only data on 8-year-olds was available for comparison at the European level. The proportion of children whose parents or caregivers filled in the family form was calculated in the whole sample (i.e., not only for 8 -year-olds).

detect and correct inaccurate information (e.g., incoherent and/or out-of-range values, outliers, etc.) using the same procedures for all countries. After this process, the country datasets were merged together for the purpose of running inter-country analyses.

\section{Statistical Analysis}

The percentage distribution of children by each variable of interest was estimated for boys and girls separately and collectively.
To determine differences in distributions by a child's sex, we employed the two-tailed Pearson $\chi^{2}$ test corrected with the Rao-Scott method. A $p$ value of 0.05 was used to define statistical significance. Data analysis was conducted at the country level and by pooling data from all countries. Pooled estimates were calculated and included one target age group per country. This was done to balance the contribution of each country to the pooled estimates, while limiting the differences in age groups included between countries. 


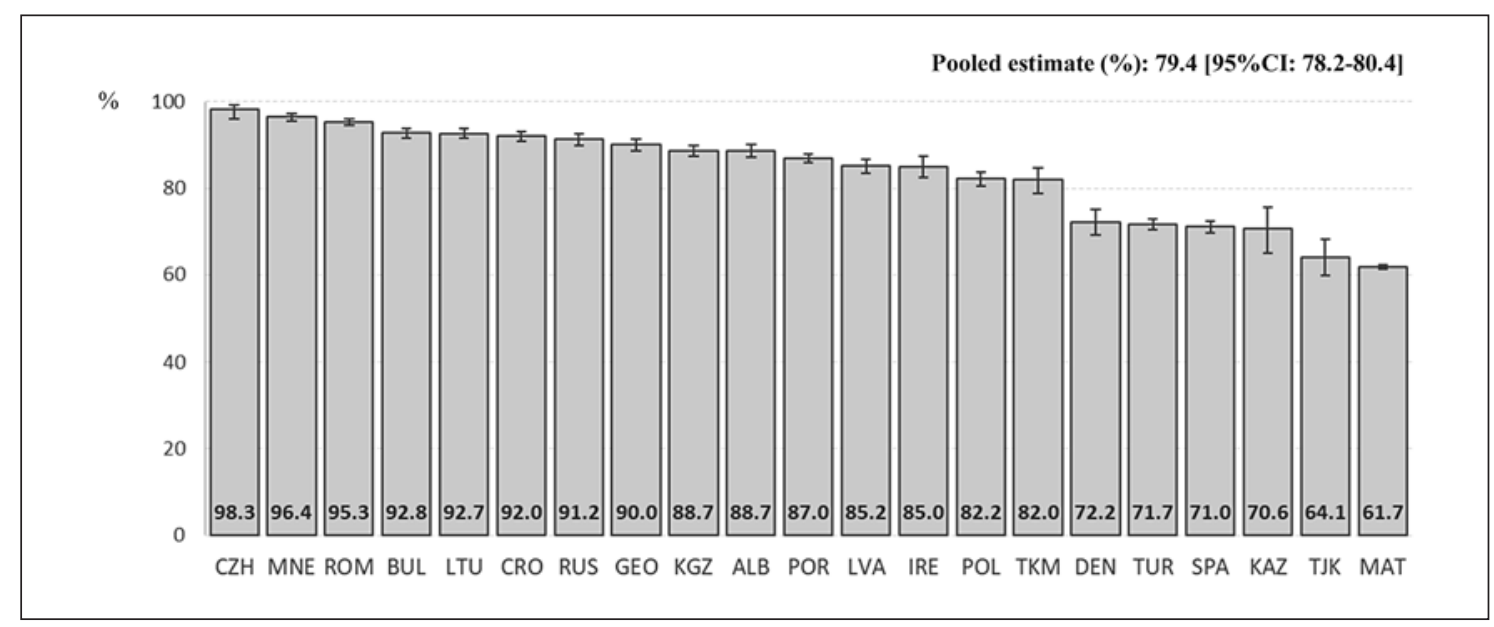

Fig. 1. Pooled and country-specific estimates (along with their $95 \%$ confidence intervals) of the percentage of children actively playing for at least $1 \mathrm{~h}$ /day (COSI/WHO Europe round 4 2015-17).

For the pooled analysis, the 7-year-old age group was used if the country targeted this age group. Otherwise, the nearest target age group was included.

Pooled estimates on PA, screen time, and sleep duration were then calculated. Data from all countries that strictly followed the COSI protocol was included in the pooled estimates, except for the data obtained in Moscow as this was not representative of the whole country.

Post-stratification weights to adjust for sampling design, oversampling, and non-response were available for all countries that applied a sampling approach; Lithuania was excluded. These weights were used in all analyses to infer the results from the sample to the population. For Lithuania, an unweighted analysis was carried out. In the pooled analysis, an adjusting factor was applied to the post-stratification weights to take into consideration differences between country population sizes. The adjusting factor was calculated based on the number of children belonging to the targeted age group according to Eurostat figures or official national statistics for 2016. All analyses took account of the complex nature of the survey (i.e., multiple stages, cluster, and stratification).

All statistical analyses were performed using the software package STATA v15.1 (StataCorp. 2017. Stata Statistical Software: Release 15. College Station, TX, USA).

\section{Results}

Table 2 shows that 150,651 children were eligible for inclusion in the analysis out of $>198,000$ invited to participate from the 25 countries. The number of children included varied between countries, from $<1,000$ in Denmark, Ireland, and San Marino to $>10,000$ in Estonia, Italy, Spain, and Turkey. These substantial differences between countries were due to differences in the number of age groups that each country targeted, the characteristics of the national school system, and a country's willingness to produce estimates at sub-national levels. There were also considerable differences between countries in the proportion of children whose parents completed the questionnaires. Turkmenistan, Italy, Tajikistan, and San Marino had the highest family participation rate (approx. 95\%), while Denmark and Ireland had the lowest (30 and $32 \%$, respectively).

\section{Active Play}

On average, around 4 in 5 children spent at least $1 \mathrm{~h} /$ day in active play. This figure varied between countries. While almost all children in Montenegro and Czechia spent at least $1 \mathrm{~h}$ /day actively playing, the corresponding figure was $<2$ out of 3 in Malta and Tajikistan (Fig. 1). In 5 countries, at least $60 \%$ spent $>2 \mathrm{~h}$ /day in active play, namely, Czechia, Kyrgyzstan, Lithuania, Montenegro, and Romania. At the other end of the scale, lower proportions were registered in Denmark, Kazakhstan, Malta, Poland, Spain, and Tajikistan, (approx. $\leq 25 \%$ ). Pooled and country-specific distributions of children by time dedicated to active play are presented in online supplementary Table 1.

\section{Sport Participation}

On average, $53.9 \%$ children were not a member of a sport or dancing club, and $30.2 \%$ spent $1-3 \mathrm{~h}$ and $15.9 \%$ spent $\geq 4 \mathrm{~h}$ per week practising sports. At the country level, these figures varied greatly. In Denmark, Latvia, Italy, and San Marino, at least $80 \%$ of children were members 


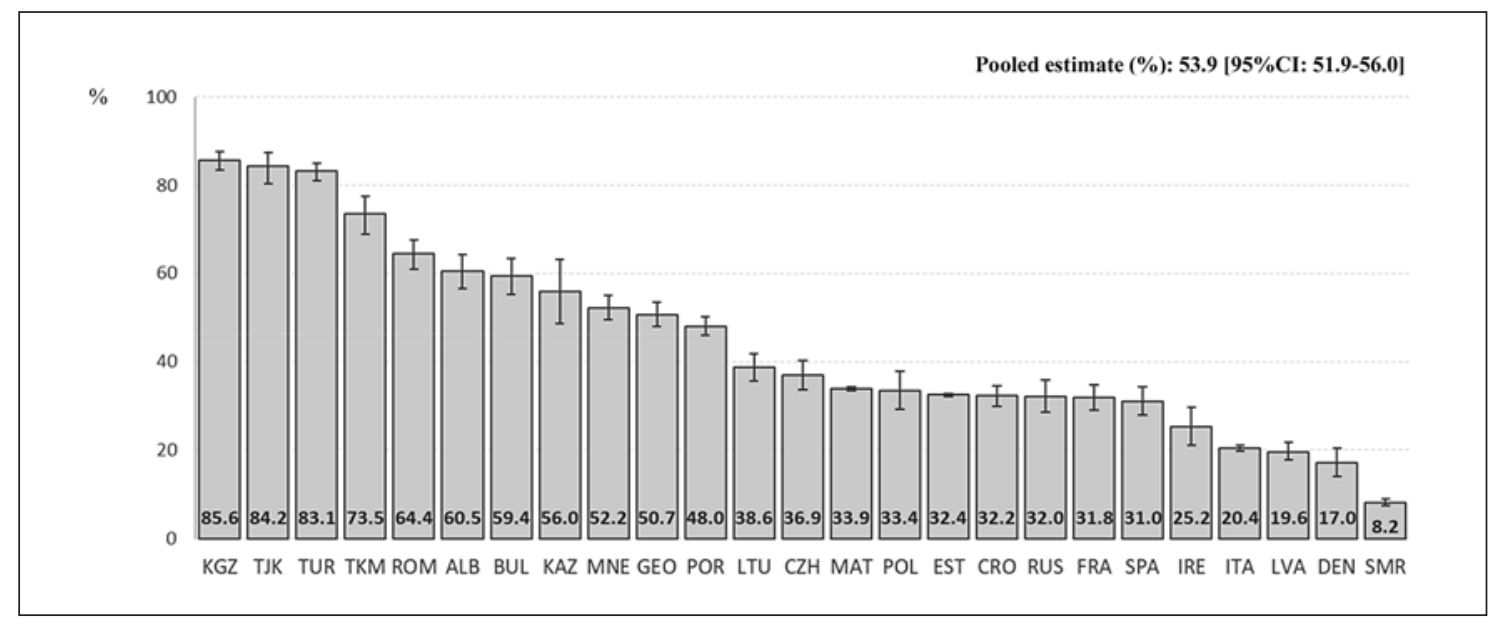

Fig. 2. Pooled and country-specific estimates (along with their $95 \%$ confidence intervals) of the percentage of children who were not members of sports/dancing clubs or did not do sports or dance at all (COSI/WHO Europe round 4 2015-17).

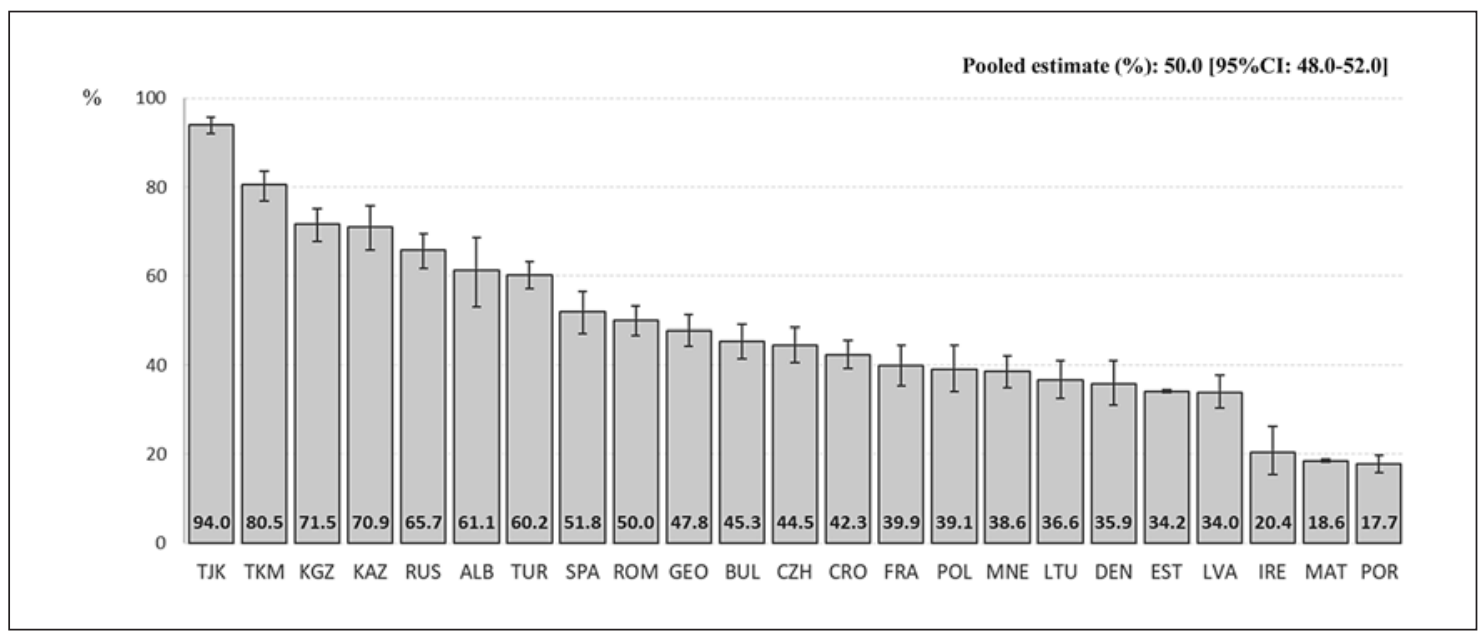

Fig. 3. Pooled and country-specific estimates (along with their $95 \%$ confidence intervals) of the percentage of children who used active transport (walking or cycling) to get to and from school (COSI/WHO Europe round 4 2015-17).

of a sport or dancing club whereas in Kyrgyzstan, Tajikistan, Turkmenistan, and Turkey, most were not $(>80 \%)$ (Fig. 2). These latter countries also recorded the lowest proportions of children practising sports for $\geq 4 /$ week $(<10 \%)$ whereas Latvia showed the highest value $(41.1 \%)$, followed by the Moscow area (36.1\%), and Croatia (33.4\%). More than 4 in 10 children practised 1-3 h/week in 6 countries, namely, Denmark, Estonia, Spain, Czechia, Malta, and Poland; all other countries showed lower proportions. Pooled and country-specific distributions of children by the time they dedicated to practising sports or dancing are presented in online Supplementary Table 2.

\section{Active Transport}

On average, 1 in 2 children used active means/transport, i.e., they walked or cycled, to get to and from school (50.0\%), and 1 in 10 used a combination of active transport and motorised vehicles. However, there were substantial differences between countries. The proportion of children using active transport ranged from most of the children in Tajiki- 


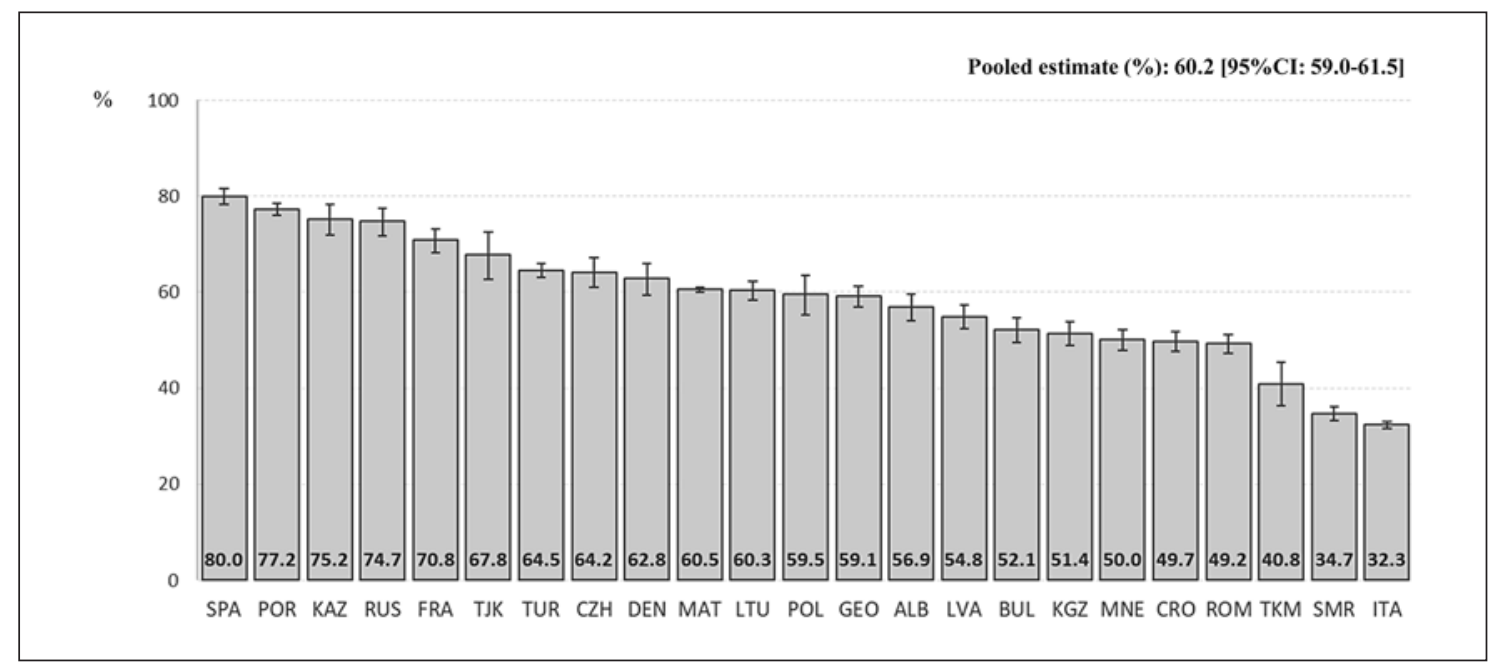

Fig. 4. Pooled and country-specific estimates (along with their $95 \%$ confidence intervals) of the percentage of children with $<2 \mathrm{~h}$ of screen time per day (COSI/WHO Europe round 4 2015-17).

stan $(94.0 \%)$ to only around $20 \%$ in Portugal, Malta, and Ireland (Fig. 3 ); in these 3 countries, most children (>70\%) travelled to school by motorised vehicle, but in Tajikistan, very few did (approx. 3\%). In the other central Asian countries, the proportion of children travelling by active transport to school was also high (80.5\% in Turkmenistan, $71.5 \%$ in Kyrgyzstan, and 70.9\% in Kazakhstan). In Croatia, Estonia, and Montenegro, around 1 in 4 children travelled to school by a combination of active transport and motorised vehicles. Pooled and country-specific distributions of children by transportation to and from school are presented in online supplementary Table 3.

\section{Screen Time}

On average, $60.2 \%$ of children were reported to be engaged in screen time for $<2 \mathrm{~h}, 25.2 \%$ for $2-3 \mathrm{~h}$, and $14.6 \%$ for $\geq 3 \mathrm{~h}$ per day, respectively. These figures varied greatly between countries. The proportion less engaged in screen time (i.e, for $<2 \mathrm{~h} /$ day) ranged from $32.3 \%$ in Italy to $80.0 \%$ in Spain (Fig. 4). Between around one-quarter (26.5\%) and more than half (58.9\%) of children had approximately $1 \mathrm{~h}$ /day of screen time and between 16.8 and $41.6 \%$ of children spent around $2 \mathrm{~h}$ /day at a screen. Pooled and country-specific distributions of children by screen time are presented in online supplementary Table 4.

\section{Sleep Duration}

On average, $84.9 \%$ of children slept for $9-11 \mathrm{~h} / \mathrm{night}$, with heterogeneity among countries. The lowest propor- tion was recorded in Ireland where half of children met the recommended number of hours of sleep per night whilst the other half slept $>11 \mathrm{~h}$ (Fig. 5). In Portugal and Spain, $>95 \%$ of children slept for $9-11 \mathrm{~h}$. On average, around $5 \%$ of children slept for $<9 \mathrm{~h}$, with country-specific values ranging from virtually none in Denmark and Ireland to $>15 \%$ in Bulgaria, Kazakhstan, Kyrgyzstan, and Tajikistan. Pooled and country-specific distributions of children by sleep duration are presented in online supplementary Table 5 .

\section{Differences by Sex}

Girls compared to boys were slightly less engaged in active play in 10 countries, with $>5$ percentage points of difference recorded between girls and boys in San Marino, Spain, and Turkey. In most of the countries, girls were less engaged in practising sports than boys. On average, a higher proportion of girls were not members of a sport or dancing club than boys ( 56.3 vs. $51.8 \%$ ), and a lower proportion $(11.8 \%)$ spent $\geq 4$ /week practising compared to boys (19.7\%). Pooled estimates and country-specific data showed no major differences in the proportions of girls and boys walking or cycling to school. In most countries, a higher proportion of boys than girls watched TV or used electronic devices for $>3 \mathrm{~h} /$ day. Pooled estimates did not show a statistically significant difference in sleep duration between boys and girls. 


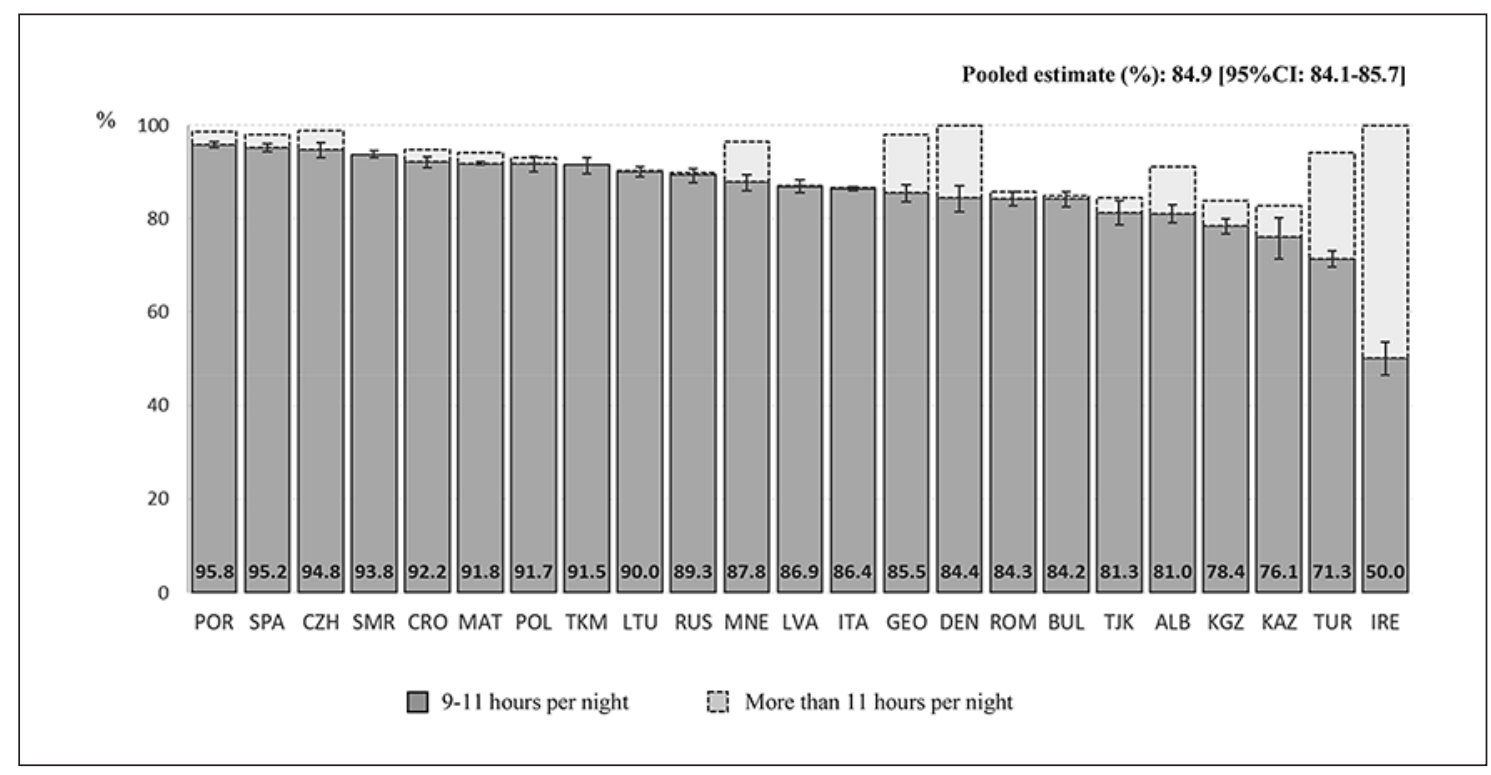

Fig. 5. Pooled and country-specific estimates (along with their $95 \%$ confidence intervals) of the percentage of children who slept for 9-11 h per night (COSI/WHO Europe round 4 2015-17).

\section{Discussion}

This study describes the prevalence of PA behaviours, screen time, and sleep duration among children aged 6-9 years from 25 countries in the WHO European Region. Comparable, nationally representative data showed pronounced differences in prevalence estimates for the various behaviours between the countries included. According to an ecological model [29], the differences observed could arise from a multitude of factors, including the policy, social, built and cultural environment, setting-specific characteristics, or differences in the natural environment.

Our results show that most children across the region engaged in active play for at least $60 \mathrm{~min} /$ day. There were variations across the countries included, which has been noted previously [30]. While active play is likely to be predominantly made up of light-intensity PA, for children in this age range active play can contribute substantially to total PA levels while reducing SB [31]. It is likely that opportunities for children to engage in active play depend on the physical environment, such as access to local parks and a recreational infrastructure [32]. The variation between the countries included in our study may also be influenced by differences in the relationship between the length of the day (the number of hours of daylight) and the weather conditions with PA levels, shown to differ between settings [33]. Different cultural values toward ac- tive play and SB may explain some variations, both between countries and for boys versus girls; it may influence determinants for PA and SB, such as parental support [34]. The lower levels of active play among girls compared to boys in certain countries could also be explained by differing socioecological influences at the levels of the family, school, and environment [35]. The effect of the social environment on PA for boys and girls in playgrounds, for example, has also been shown to contribute to these gender differences in relation to active play [36].

Depending on the national context, sports organisations may function as an essential facilitator for PA and sport participation among children. Across the region, we found that around half of both boys and girls were members of a sport or dancing club; this was lower than the prevalence of sport participation found in similar studies $[30,37]$. However, the COSI questionnaire asks specifically about membership of a sports or dancing club rather than participation, and this could explain why the prevalence we recorded was lower than in other studies that also captured informal sports participation (especially in Eastern Europe and Central Asia where formal grassroot sports organisations are less common). The proportion of girls participating in sport for $\geq 4 \mathrm{~h}$ /week was generally around half that of boys; this may be due to a lack of a variety of activities to better suit the preferences of girls [38].
Whiting et al. 
Active transport to school can produce important health benefits [39]. There was substantial variation between countries in the prevalence of children actively travelling to school, which aligns with previous findings [30]. In the countries of Central Asia, where active transport may be a necessity, it was shown that $>3$ out of 4 children walked or cycled to school; in Portugal and Malta, where travelling to school by car may be more of a cultural norm, this proportion was $<1$ out of 5 . This has also been noted previously [30]. Other factors influencing whether children walk, cycle, use public transport, or are driven to school by car are: the length of the school day and timetable, the availability of safe walking or cycling paths, access to free public transport, the location of elementary schools within communities, road safety, and weather conditions [32].

It is recommended that children in this age group spend $<2 \mathrm{~h} /$ day engaging in recreational screen to avoid negative health outcomes [19-21]. Across the region, we found that more than half the children met this recommendation, which was much higher than recorded by previous studies that used self-reporting [40] and objective methods [41] to estimate screen time. It has been noted previously that parents underestimate screen time compared to children's self-reports [42], as they may be unaware of how much their children are using screenbased devices throughout the entire day. As with the other behaviours measured in our study, the differences between countries may have been due to variations in social/cultural and physical environments which are important in determining SB among children [43]. Across the region, longer screen time per day was more prevalent among boys than girls, in line with previous findings [44].

Sleep plays a vital role in health. It is recommended that children aged 6-9 years sleep for 9-11 h/night [1921 ], and short sleep duration can be detrimental to physical and mental health and well-being [45]. We found that, across the region, most children achieved the recommended 9-11 h of sleep, a much higher proportion than found in other studies that used objective measurements to estimate sleep duration [40]. There were substantial differences between countries, which aligns with previous findings where children in more northerly countries generally slept the longest, likely due to different cultural and environmental characteristics [46]. For some of the countries that did not meet the recommendations, short sleep duration was not the issue; rather, the lower prevalence was due to the higher proportion of children recorded as sleeping for $>11 \mathrm{~h} /$ night.

A key strength of this study is the use of systematic, nationally representative data collected via the COSI sys- tem. In many of the countries included, these are the firstever national estimates available of PA behaviours, screen time, and sleep duration of school-aged children. Another strength is the large sample size which increases confidence in the findings and limits the impact of outliers. There are, however, some limitations which must be mentioned. Due to the way the variables were operationalised, we were unable to calculate total PA according to WHO recommendations by combining active transport, active play, and sport participation. There may also be substantial discrepancies between the results of parent/ caregiver reporting and other methods of measuring PA, screen time, and sleep, meaning that all results should be viewed with caution. Reporting of PA and SB might also have been vulnerable to differences in the terminology used across countries to describe these behaviours [47, 48]; the social desirability and attitudes related to higher PA levels and SB among children may also have varied across countries and influenced the responses. Finally, there is potential for selection bias, with the families that allowed their children to participate and were willing to complete the family form not necessarily representing those that did not give their consent.

\section{Conclusion}

An analysis of the results of the WHO COSI 2015-2017 study provides a unique overview of the PA behaviours, screen time, and sleep duration of children aged 6-9 years in the WHO European Region. The heterogeneity in the prevalence between countries shows that there are opportunities for national policymakers to learn from experiences across the region and adopt what seems to be working. These results can help countries to prioritise actions to address specific PA behaviours that may be more effective in increasing overall PA levels and reducing SB. Local action is needed to address the lack of engagement in PA and the overuse of screen-based devices as well as to ensure the benefits of enough sleep. Further development of national procedures for monitoring these and other risk factors for NCDs are needed. Evaluations of existing prevention and management strategies targeting young people could be utilised to design and implement more effective interventions.

\section{Acknowledgements}

We gratefully acknowledge all participating children, and the parents, schoolteachers, and school principals for kindly volunteering to collaborate in the study. We also thank the examiners 
and regional and local supervisors/coordinators who collected the data in each country. Furthermore, we would like to acknowledge the contribution of other researchers and/or principal investigators who contributed to the COSI study or the interpretation of the results, namely: Bianca Fuchs-Nehold, Adelheid Weber, Isabelle Moyersoen, Marie Kunesova, Päivi Mäki, Tiina Laatikainen, Valikhan Akhmetov, Dorothy Zammit, Svetlana Cociu, Constanta Huidumac, Elena Bogova, Elena Sacchini, Višnja Đorđić, Britt Eriksson, Sanavba Rakhmatulloeave, María Ángeles Dal Re, Dilorom Akhmedova, Barno Abdusamatova, Benoit Salanave, Julie Malherbe, Else Karin Grøholt, Natalia Silitrari, Vladislav Zbanatsky, Dilorom Akhmedova, Tue Kristensen, Jolanda Boer, and Andrea Horvath.

\section{Statement of Ethics}

The WHO COSI study protocol was approved by international ethics guidelines for biomedical research involving human subjects, and all procedures were also approved by local ethics committees in each country. Furthermore, the children's parents or guardians gave their written informed consent.

Ethics approval was provided by the following countries: Albania (Scientific Committee of Institute of Public Health, decision No. 953, 13/07/2015), Bulgaria (Commission of Medical Ethics at the National Center of Public Health and Analyses, Sofia, Bulgaria, Project identification code - 060 - MП 325 -68 COSI, 25.02.2016), Croatia (Ethics Committee of the Croatian Institute for Public Health, registry No. 80-2660/1-15, 25.9.2015), Czechia (Ethics Committee of the Institute of Endocrinology, Prague, Czech Republic, AZV MZČR 17-31670 A, 20/06/2016), Denmark (Research and Innovation Organization, SDU, 10.829, 27/06/2016), Estonia (Tallinn Medical Research Ethics Committee, TMEK decision No. 1376, 28/03/2016), Georgia (Bioethics Council at National Center for Disease Control and Public Health of Georgia, project identification code: 2019-52, 4 November, 2019), Ireland (University College Dublin Human Research Ethics Committee - Sciences, Project identification code: LS-15-43-Heinen-Kelleher, date of approval: 26 August 2015), Italy (National Institute of Health, Prot. PRE - 739/15, 10 November 2015), Kazakhstan (Scientific and technical program "Development and implementation of modern technologies for healthy lifestyle promotion and prevention of diseases based on the study of non-medical determinants of health among children," 2015), Kyrgyzstan (The Ethics Committee on compliance of research to ethical norms for medical research, project identification code: No. 1/1, Date of approval: 22 February 2018), Latvia (Central Medical Ethics Committee, project identification code: 01-29.1/6, date of approval: 25.09.2015), Lithuania (Lithuanian Bioethics Committee (Lietuvos bioetikos komitetas); project identification code: 08-02-19; 19 February, 2008. After the approval we received renewal of a bioethics authorization in 2010 (on 2010-01-04), 2013 (on 2013-01-09) and 2019 (on 2019-03-12), Montenegro (Ethics Committee of the Institute of Public Health of Montenegro Project identification code: WHO 2016/627456-0, date of approval: 28th April 20), Poland (Bioethics Committee of the Institute of Mother and Child, Warsaw, Poland, project identification No. 22/2015, date of approval: 26 November 2015), Portugal (National Commission of Data Protection; Aut $n^{\circ} 5418 / 2016$ for all rounds of COSI Portugal, 7 June 2016), Romania (Intern Ethics Committee of the National Institute of Public
Health, Romania , project identification code: WHO 2016/6503010, date of approval: 6 April 2016), Russian Federation (National Institute of Health., Prot. PRE - 739/15, November 2015 National Institute of Health, Prot. PRE - 739/15, November 2015), Tajikistan (Ministry of Health and Social Protection of Tajikistan, project identification code: \#858, date of approval: 18 November 2016), Turkey (Kecioren Training and Research Hospital, Clinical Researches Ethics Committee; approval date: 26.10.2016, project identification code: Health System Strengthening and Support Project, LN: 8531-TR, date of approval 2015: L.2.12. Obesity Fighting Project, subcomponent 1.1, L.2.11). In Turkmenistan, ethics approval was granted from the Ministry of Healthcare and Medical Industry (MOHMI). Malta did not go through an ethics committee as all the work involved in COSI data collection and analysis is part and parcel of the existing School Health Service, which is an ongoing process. Data for COSI in Spain was collected as part of the ALADINO Spanish study, which did not ask for an ethics committee approval, since this is not mandatory in Spain. However, the principal investigators confirm that the study was conducted in accordance with the Declaration of Helsinki and all parents/guardians of subjects participating in the 4 rounds gave their informed consent for inclusion before they participated in the study.

\section{Conflict of Interest Statement}

The authors declare no conflicts of interest. The funders had no role in the design of the study, collection, analyses, or interpretation of data, writing of the manuscript, or the decision to publish the results.

The writing group takes sole responsibility for the content of this article, and the content of this article reflects the views of the authors only. S.W, I.R. J.B. and J.W. are staff members of the WHO and M.B. and R.M. are WHO consultants. The WHO is not liable for any use that may be made of the information contained herein.

\section{Funding Sources}

The authors gratefully acknowledge support from a grant from the Russian Government in the context of the WHO European Office for the Prevention and Control of NCDs.

Data collection in the following countries was made possible through funding. Albania: WHO through the Joint Programme on Children, Food Security and Nutrition "Reducing Malnutrition in Children" (the Millennium Development Goals Achievement Fund) and the Institute of Public Health; Bulgaria: Ministry of Health, National Centre of Public Health and Analyses, WHO Regional Office for Europe; Croatia: Ministry of Health, Croatian Institute of Public Health and WHO Regional Office for Europe; Czechia: grants AZV MZČR 17-31670 A and MZČR - RVO EÚ 00023761; Denmark: Danish Ministry of Health; Estonia: Ministry of Social Affairs, Ministry of Education and Research (IUT 42-2), WHO Country Office, and National Institute for Health Development; France: Sante Publique France, the French Agency for Public Health; Georgia: WHO; Ireland: Health Service Executive; Italy: Ministry of Health and Italian National Institute of Health; $\mathrm{Ka}$ zakhstan: Ministry of Health of the Republic of Kazakhstan and WHO Country Office; Kyrgyzstan: WHO; Latvia: Ministry of 
Health, Centre for Disease Prevention and Control; Lithuania: Science Foundation of Lithuanian University of Health Sciences and Lithuanian Science Council and WHO; Malta: Ministry of Health; Montenegro: WHO and Institute of Public Health of Montenegro; Poland: National Health Programme, Ministry of Health; Portugal: Ministry of Health Institutions, the National Institute of Health, Directorate General of Health, Regional Health Directorates and the kind technical support from the Center for Studies and Research on Social Dynamics and Health (CEIDSS); Romania: Ministry of Health; San Marino: Health Ministry, Educational Ministry, Social Security Institute and Health Authority; Spain: Spanish Agency for Food Safety and Nutrition (AESAN); Turkmenistan: WHO Country Office in Turkmenistan and Ministry of Health; Turkey: Turkish Ministry of Health and the World Bank.

\section{Author Contributions}

S.W., M.B. and J.B. conceptualized the manuscript. S.W., M.B., M.P., K.A.O., and P.G. drafted the manuscript. M.B. conducted all analyses. J.B. made substantial contribution to drafts of both the manuscript and the COSI study protocol as well as interpretation of the results. J.H., V.D., S.M.M., H.Z., T.H., E.N., L.S., C.C.K., M.H., A.S., P.N., A.A., S.A., G.A., Z.U., I.P., A.P., V.F.S., E.K., S.P., A.F., A.R., M.A.C., L.A.B., V.P., A.G., M.G.S., E.G.G., Z.A., N.Y. and M.T. commented on drafts of the paper and contributed with data collection and data cleaning. D.W., K.S., D.S., A.F.H., E.M.I., M.H., V.A.K., S.O., L.T., G.S., K.R.J., I.S., J.W., I.R., R.M., H.R., A.S.F., I.H.B., M.R. and W.A. contributed to drafts of the manuscript. All authors contributed to and approved the paper.

\section{References}

1 WHO. Global recommendations on physical activity for health. Geneva: World Health Organization; 2010.

2 Kuzik N, Carson V, Andersen LB, Sardinha LB, Grøntved A, Hansen BH, et al.; International Children's Accelerometry Database (ICAD) Collaborators. Physical Activity and Sedentary Time Associations with Metabolic Health Across Weight Statuses in Children and Adolescents. Obesity (Silver Spring). 2017 Oct;25(10):1762-9.

3 Moore JB, Beets MW, Brazendale K, Blair SN, Pate RR, Andersen LB, et al. Associations of Vigorous-Intensity Physical Activity with Biomarkers in Youth. Med Sci Sports Exerc. 2017 Jul;49(7):1366-74.

4 Freedman DS, Khan LK, Serdula MK, Dietz WH, Srinivasan SR, Berenson GS. The relation of childhood BMI to adult adiposity: the Bogalusa Heart Study. Pediatrics. 2005 Jan; 115(1):22-7.

5 Li C, Ford ES, Zhao G, Mokdad AH. Prevalence of pre-diabetes and its association with clustering of cardiometabolic risk factors and hyperinsulinemia among U.S. adolescents: National Health and Nutrition Examination Survey 2005-2006. Diabetes Care. 2009 Feb; 32(2):342-7.

6 Schwimmer JB, Burwinkle TM, Varni JW. Health-related quality of life of severely obese children and adolescents. JAMA. 2003 Apr; 289(14):1813-9.

7 Janicke DM, Marciel KK, Ingerski LM, Novoa W, Lowry KW, Sallinen BJ, et al. Impact of psychosocial factors on quality of life in overweight youth. Obesity (Silver Spring). 2007 Jul;15(7):1799-807.

8 Tsiros MD, Olds T, Buckley JD, Grimshaw P, Brennan L, Walkley J, et al. Health-related quality of life in obese children and adolescents. Int J Obes. 2009 Apr;33(4):387-400.

9 Brixval CS, Rayce SL, Rasmussen M, Holstein BE, Due P. Overweight, body image and bullying-an epidemiological study of 11- to 15-years olds. Eur J Public Health. 2012 Feb; 22(1):126-30.
10 Dietz WH, Robinson TN. Clinical practice. Overweight children and adolescents. N Engl J Med. 2005 May;352(20):2100-9.

11 Kristensen PL, Møller NC, Korsholm L, Wedderkopp N, Andersen LB, Froberg K. Tracking of objectively measured physical activity from childhood to adolescence: the European youth heart study. Scand J Med Sci Sports. 2008 Apr;18(2):171-8.

12 Telama R. Tracking of physical activity from childhood to adulthood: a review. Obes Facts. 2009;2(3):187-95.

13 WHO. Global Action Plan for Physical Activity 2018-2030: more active people for healthier world. Geneva: WHO; 2019.

14 World Health Organization, Regional Office for Europe. Physical activity strategy for the WHO European Region 2016-2025. Copenhagen: World Health Organisation Regional Office for Europe; 2016.

15 Pearson N, Biddle SJ. Sedentary behavior and dietary intake in children, adolescents, and adults. A systematic review. Am J Prev Med. 2011 Aug;41(2):178-88.

16 Miller MA, Kruisbrink M, Wallace J, Ji C, Cappuccio FP. Sleep duration and incidence of obesity in infants, children, and adolescents: a systematic review and meta-analysis of prospective studies. Sleep (Basel). 2018 Apr; 41(4). https://doi.org/10.1093/sleep/ zsy018.

17 Sparano S, Lauria F, Ahrens W, Fraterman A, Thumann B, Iacoviello L, et al. Sleep duration and blood pressure in children: analysis of the pan-European IDEFICS cohort. J Clin Hypertens (Greenwich). 2019 May; 21(5):572-8.

18 Thumann BF, Börnhorst C, Michels $\mathrm{N}$, Veidebaum T, Solea A, Reisch L, et al.; IDEFICS and I.Family consortia. Cross-sectional and longitudinal associations between psychosocial well-being and sleep in European children and adolescents. J Sleep Res. 2019 Apr;28(2):e12783.

19 Tremblay MS, Carson V, Chaput JP, Connor Gorber S, Dinh T, Duggan M, et al. Canadian
24-Hour Movement Guidelines for Children and Youth: An Integration of Physical Activity, Sedentary Behaviour, and Sleep. Appl Physiol Nutr Metab. 2016 Jun;41(6 Suppl 3):S311-27.

20 Department of Health. Australian Government. Australian 24-Hour Movement Guidelines for Children and Young People (5-17 years): an integration of physical activity, sedentary behaviour and sleep. Canberra: Department of Health; 2019.

21 Ministry of Health, New Zealand Government. Physical Activity Guidelines for Children and Young People. Wellington: Ministry of Health, New Zealand Government; 2017.

22 WHO Regional Office for Europe. WHO European Childhood Obesity Surveillance Initiative. Data collection procedures, 2016. Copenhagen: WHO/Europe; 2016.

23 Wijnhoven T, Branca F. WHO European Childhood Obesity Surveillance Initiative. Protocol, version January 2008. Copenhagen: WHO/Europe; 2008.

24 WHO Regional Office for Europe. WHO European Childhood Obesity Surveillance Initiative. Protocol, version August 2010. Copenhagen: WHO/Europe; 2010.

25 WHO Regional Office for Europe. WHO European Childhood Obesity Surveillance Initiative. Protocol, version October 2012. Copenhagen: WHO/Europe; 2012.

26 WHO Regional Office for Europe. WHO European Childhood Obesity Surveillance Initiative. Protocol, 2016. Copenhagen: WHO/ Europe; 2016.

27 World Health Organization, Council for International Organizations of Medical Sciences. International ethical guidelines for healthrelated research involving humans. Geneva: CIOMS; 2017.

28 Spinelli A, Buoncristiano M, Kovacs VA, Yngve A, Spiroski I, Obreja G, et al. Prevalence of Severe Obesity among Primary School Children in 21 European Countries. Obes Facts. 2019;12(2):244-58. 
29 Sallis JF, Cervero RB, Ascher W, Henderson KA, Kraft MK, Kerr J. An ecological approach to creating active living communities. Annu Rev Public Health. 2006;27(1):297-322.

30 Tremblay MS, Gray CE, Akinroye K, Harrington DM, Katzmarzyk PT, Lambert EV, et al. Physical activity of children: a global matrix of grades comparing 15 countries. J Phys Act Health. 2014 May;11(s1 Suppl 1):S11325.

31 Brockman R, Jago R, Fox KR. The contribution of active play to the physical activity of primary school children. Prev Med. 2010 Aug;51(2):144-7.

32 Davison KK, Lawson CT. Do attributes in the physical environment influence children's physical activity? A review of the literature. Int J Behav Nutr Phys Act. 2006 Jul;3(1):19.

33 Harrison F, Goodman A, van Sluijs EM, Andersen LB, Cardon G, Davey R, et al.; on behalf the ICAD collaborators. Weather and children's physical activity; how and why do relationships vary between countries? Int J Behav Nutr Phys Act. 2017 May;14(1):74.

34 Sallis JF, Prochaska JJ, Taylor WC. A review of correlates of physical activity of children and adolescents. Med Sci Sports Exerc. 2000 May;32(5):963-75.

35 Telford RM, Telford RD, Olive LS, Cochrane T, Davey R. Why Are Girls Less Physically Active than Boys? Findings from the LOOK Longitudinal Study. PLoS One. 2016 Mar; 11(3):e0150041.
36 Reimers AK, Schoeppe S, Demetriou Y, Knapp G. Physical Activity and Outdoor Play of Children in Public Playgrounds-Do Gender and Social Environment Matter? Int J Environ Res Public Health. 2018 Jun;15(7):1356.

37 Kokko S, Martin L, Geidne S, Van Hoye A, Lane A, Meganck J, et al. Does sports club participation contribute to physical activity among children and adolescents? A comparison across six European countries. Scand J Public Health. 2019 Dec;47(8):851-8.

38 Karsten L. Children's Use of Public Space: The Gendered World of the Playground. Childhood. 2003 Nov; 10(4):457-73.

39 Larouche R, Saunders TJ, Faulkner G, Colley $\mathrm{R}$, Tremblay M. Associations between active school transport and physical activity, body composition, and cardiovascular fitness: a systematic review of 68 studies. J Phys Act Health. 2014 Jan;11(1):206-27.

40 Roman-Viñas B, Chaput JP, Katzmarzyk PT Fogelholm M, Lambert EV, Maher C, et al.; ISCOLE Research Group. Proportion of children meeting recommendations for 24-hour movement guidelines and associations with adiposity in a 12-country study. Int J Behav Nutr Phys Act. 2016 Nov;13(1):123.

41 Atkin AJ, Sharp SJ, Corder K, van Sluijs EM; International Children's Accelerometry Database (ICAD) Collaborators. Prevalence and correlates of screen time in youth: an international perspective. Am J Prev Med. 2014 Dec; 47(6):803-7.

42 Wood CT, Skinner AC, Brown JD, Brown CL, Howard JB, Steiner MJ, et al. Concordance of Child and Parent Reports of Children's Screen Media Use. Acad Pediatr. 2019 Jul;19(5):52933.
43 Hidding LM, Altenburg TM, van Ekris E, Chinapaw MJ. Why Do Children Engage in Sedentary Behavior? Child- and Parent-Perceived Determinants. Int J Environ Res Public Health. 2017 Jun;14(7):E671.

44 LeBlanc AG, Broyles ST, Chaput JP, Leduc G, Boyer C, Borghese MM, et al. Correlates of objectively measured sedentary time and selfreported screen time in Canadian children. Int J Behav Nutr Phys Act. 2015 Mar;12(1):38.

45 Chaput JP, Gray CE, Poitras VJ, Carson V, Gruber R, Olds T, et al. Systematic review of the relationships between sleep duration and health indicators in school-aged children and youth. Appl Physiol Nutr Metab. 2016 Jun; 41(6 Suppl 3):S266-82.

46 Hense S, Barba G, Pohlabeln H, De Henauw S, Marild S, Molnar D, et al. Factors that influence weekday sleep duration in European children. Sleep (Basel). 2011 May;34(5): 633-9.

47 Tremblay MS, Aubert S, Barnes JD, Saunders TJ, Carson V, Latimer-Cheung AE, et al.; SBRN Terminology Consensus Project Participants. Sedentary Behavior Research Network (SBRN) - Terminology Consensus Project process and outcome. Int J Behav Nutr Phys Act. 2017 Jun;14(1):75.

49 Thivel D, Tremblay A, Genin PM, Panahi S, Rivière D, Duclos M. Physical Activity, Inactivity, and Sedentary Behaviors: Definitions and Implications in Occupational Health. Front Public Health. 2018 Oct;6:288. 